\title{
Review of: "A novel regulatory gene promotes novel cell fate by suppressing ancestral fate in the sea anemone Nematostella vectensis"
}

\author{
Grigory Genikhovich ${ }^{1}$ \\ 1 Universität Vienna
}

Potential competing interests: The author(s) declared that no potential competing interests exist.

The question how novelty is generated is one of the most exciting in the evolutionary developmental biology. In Cnidaria, the best-known novelty is the cnidocyte. In their new preprint, Babonis et al. demonstrate an interesting example of a putative cnidarian-specific Zn-finger transcription factor, ZNF845, which appears to act as a switch between the RF-amide positive sensory cell fate and the cnidocyte fate in the sea anemone Nematostella vectensis. They characterize the expression of ZNF845, show that it acts downstream of SoxB2, and demonstrate that the suppression of the ZNF845 expression by RNAi or morpholino-mediated knockdown leads to a strong reduction in the cnidocyte numbers at the planula stage, downregulation of a number of cnidocyte marker genes and, conversely, to an upregulation of the sensory neuron marker $R F$-amide. They further show that ZNF845 may be acting through one of the NR2F paralogs, NR12, which appears to prevent the differentiation of the future cnidocytes into RF-amide positive neurons. NR12 is expressed in a subset of ZNF845-expressing cells, its expression is reduced upon ZNF845 knockdown, and NR12 knockdown resulted in a significant increase of RF-amide expressing cells without affecting cnidocyte numbers. The rest of the paper is devoted to tracing the evolution of the $\mathrm{Zn}$ finger transcription factors and summarizes the views of the authors on the process of cell type evolution. While I am very impressed by the new results, there are several points the authors might want to consider addressing either prior to sending these data to a journal or in their future work.

1. There is no explanation why and how ZNF845 was chosen as a potentially interesting gene to analyze with regard to the mechanism of suppression of the neural phenotype in cnidocytes. Was this part of some massive screen?

2. Fig. S1 shows that ZNF845 expression upon RNAi recovers by late planula, but the authors do not comment on the reasons for this. Do they also observe this quick recovery in the morphants? Do primary polyps developing from the KD planulae have normal cnidocytes? It would be important to know whether the putative role of ZNF845 in committing the cells to the cnidocyte fate is restricted to the early developmental stages. Ultimately, this, of course, would require generating and characterizing a mutant.

3. According to the manuscript, ZNF845 can be expressed in EdU positive and in SoxB2 positive cells, and ZNF845 knockdown increases the number of RF-amide positive neurons. POU4 is expressed in 
postmitotic cells, which stopped expressing SoxB2 but can express RF-amide and Ncol3. This probably puts them downstream of ZNF845 (in line with the qPCR data presented in the preprint). Is that so? Can ZNF845 expressing cells express RF-amide?

4. The authors say that in order to understand how ZNF845 suppresses RF-amide expression they searched for inhibitory TFs co-expressed with the cnidocyte-specific genes. I would be interested to know what the search strategy was. What made the authors think that this regulation was not direct but via an intermediate TF? And why did they look at the NR2F genes in particular? My guess would be that there are dozens if not hundreds of potential transcriptional repressors in the cnidocyte transcriptomes.

5. NR11, NR12, and NR13 are expressed in different subpopulations of cnidocytes. How did the authors select NR12? What about the knockdowns of the NR11 and NR13, which appear to be much stronger influenced by the ZNF845 knockdown and, as they show, are not co-expressed with NR12? Do their KDs also affect the number of the RF-amide neurons?

6. If $N R 12$ is the ultimate repressor of RF-amide expression, how does it affect POU4 expression (if at all)? How does POU4 KD affect NR12?

7. If NR12 prevents cnidocytes from becoming RF-amide positive neurons, how do you explain the lack of change in the number of cnidocytes upon NR12 knockdown, although the number of RF-amide positive cells increases? It does not look like NR12 works as a binary switch in this context.

8. It would be fantastic to test whether NR12 overexpression rescues the ZNF845 KD phenotype. This is, of course, a hugely challenging experiment to make and can be a whole PhD project just by itself.

9. The last part of the Results called "Modeling cell type expansion" is entirely theoretical. In my opinion, it either belongs in the Discussion or (together with the current Discussion) - in a separate review article. The Discussion currently appears quite "detached" from the actual results of the paper. My suggestion would be to focus on discussing the experimental findings of the paper.

In spite of all these questions, I would like to stress that this is a really interesting work, and a nice example of a novel gene responsible for generating a novel cell type. 